\title{
Diagnosis and Management of Primary Prostatic Signet Ring Cell Carcinoma: Single Center Experience
}

\section{Min Tang ( $\sim$ mintang@njmu.edu.cn )}

The First Affiliated Hospital of Nanjing Medical University https://orcid.org/0000-0002-8350-5148

\section{Pu Li}

The First Affiliated Hospital of Nanjing Medical University

\section{Zihan Zhou}

Department of First Clinical Medical College of Nanjing Medical University

\section{Meiling Bao}

The First Affiliated Hospital of Nanjing Medical University

Jie Li

The First Affiliated Hospital of Nanjing Medical University

\section{Xiaoxin Meng}

The First Affiliated Hospital of Nanjing Medical University

\section{Bianjiang Liu}

The First Affiliated Hospital of Nanjing Medical University

\section{Research Article}

Keywords: Primary prostatic signet ring cell carcinoma, diagnosis, treatment, prognosis

Posted Date: August 14th, 2021

DOl: https://doi.org/10.21203/rs.3.rs-803581/v1

License: (c) (i) This work is licensed under a Creative Commons Attribution 4.0 International License. Read Full License 


\title{
Diagnosis and management of primary prostatic signet ring cell carcinoma : Single center experience
}

\author{
$\mathrm{Pu} \mathrm{Li}^{1 \#}$, Zihan Zhou ${ }^{2 \#}$, Meiling Bao ${ }^{3 \#}, \mathrm{Jie} \mathrm{Li}^{1}$, Xiaoxin Meng ${ }^{1}$, Bianjiang Liu ${ }^{1 *}$, Min Tang ${ }^{1 *}$ \\ ${ }^{1}$ Department of Urology, The First Affiliated Hospital of Nanjing Medical University, Nanjing, China. \\ ${ }^{2}$ Department of First Clinical Medical College of Nanjing Medical University, Nanjing, China. \\ ${ }^{3}$ Department of Pathology, The First Affiliated Hospital of Nanjing Medical University, Nanjing, \\ China.
}

\# These authors contributed equally to this work

Correspondence to: Min Tang; Bianjiang Liu. Department of Urology, The First Affiliated Hospital of Nanjing Medical University, No. 300 Guangzhou Road, Nanjing, Jiangsu, 210000, China. Tel: +8625 6830 3176, Fax: +86 256830 3176, Email: mintang@njmu.edu.cn; bjliu@njmu.edu.cn

\begin{abstract}
Acknowledgements : This work was supported by the National Natural Science Foundation of China (81600514), Jiangsu Province Postdoctoral Research Support Project (2021K595C), Jiangsu Science and Technology Association Young Science and Technology Talents Lifting Project.
\end{abstract}

Authors' Contributions: All authors contributed to the study conception and design. Material preparation, data collection and analysis were performed by $\mathrm{Pu} \mathrm{Li}$, Zihan Zhou, and Meiling Bao. The first draft of the manuscript was written by $\mathrm{Pu} \mathrm{Li}$ and all authors commented on previous versions of the manuscript. All authors read and approved the final manuscript. 


\section{Abstract}

2 Background: Primary prostatic signet ring cell carcinoma (PPSRCC) is an extremely rare malignant

3 tumor without definitive treatment approach. Herein, we retrospectively summarized the experience of

4 diagnosis and management of ten PPSRCC cases.

5 Methods: A total of ten PPSRCC patients were included in this study definitely diagnosed by postoperative pathology at the First Affiliated Hospital of Nanjing Medical University from November 2001 to December 2020. Clinical characteristics, image features, therapeutic procedures, histological diagnosis and outcomes of them were retrospectively analyzed.

9 Results: Characteristics of the patient population were ten males with age of $67.8 \pm 7.2$ years. Among them, five cases were asymptomatic and five were manifested as dysuria. All ten patients received PSA examination preoperatively. Nine patients of ten accepted multiparametric MRI due to elevated PSA value, and further adopted prostatic biopsy. Among them, five patients were diagnosed as prostatic adenocarcinoma, and the other four cases were found a mixture of SRCC and adenocarcinoma. The above nine patients accepted laparoscopic or robot-assisted radical prostatectomy. Only one patient with normal PSA value adopted TURP. Postoperatively pathological results confirmed SRCC mixed with prostatic adenocarcinoma in nine cases, and only one patient with pure SRCC. After surgery, nine patients received adjuvant hormone therapy, one of which accepted radiotherapy simultaneously. The other one patient with pure SRCC didn't accept any adjuvant therapy. None of the ten cases had gastrointestinal tumor history. During a mean follow-up of 43.6 months, eight patients were alive without obvious disease progression.

Conclusions: In summary, PPSRCC is a rare malignant tumor with few early symptoms, highly malignancy, rapid disease progression and poor prognosis. PPSRCC is frequently accompanied by high 
41 Methods

42 survival rate.

\section{(}

\section{Patients}

grade prostate adenocarcinoma patterns. Early diagnosis and timely radical resection of prostate cancer, endocrine therapy, radiotherapy and chemotherapy may improve the prognosis.

Keywords: Primary prostatic signet ring cell carcinoma; diagnosis; treatment; prognosis

\section{Introduction}

Signet ring cell carcinoma (SRCC) is a specific type of mucinous adenocarcinoma that occurs mostly in the gastrointestinal tract. Primary prostatic signet ring cell carcinoma (PPSRCC) is rare and highly malignant. Uyama first described this disease in 1979 [1]. So far, about 110 cases have been reported in relevant literatures, accounting for about $2.5 \%$ of prostate adenocarcinoma [2]. Most of the patients are middle-aged and elderly men. The clinical symptoms of PPSRCC are similar to those of typical poorly differentiated prostate cancer, mainly including frequent urination, urgent urination, lower abdominal pain, dysuria and other symptoms. Early diagnosis and combined treatment may improve the long-term

Herein, we retrospectively analyzed the clinical manifestation, therapeutic procedures, histological diagnosis and outcomes of ten PPSRCC cases treated in our center from 2001 to 2021. Moreover, we performed a brief literature review to the diagnosis and management of PPSRCC to provide a comprehensive understanding of this uncommon tumor.

Ten patients with signet ring cell carcinoma of prostate were diagnosed by postoperative pathology in the First Affiliated Hospital of Nanjing Medical University from November 2001 to December 
2020.We retrospectively reviewed our experience in diagnosis and treatment. We summarized and compared the age, clinical symptoms, radiographic findings, pathologic findings, surgical methods, and outcomes of these patients. All procedures carried out in this study were in line with the Declaration of Helsinki (Revised 2013) and this study was approved by the Ethics Committee of the First Affliated Hospital of Nanjing Medical University (No. 2020-SR-148) and written informed consent for publication of the patients' information and images was entirely obtained.

\section{Imaging and pathological examination}

All the ten patients received PSA test, as well as multiparametric MRI (mpMRI). Prostate needle biopsy was adopted in nine cases preoperatively. Postoperative routine histopathology and immunohistochemistry were used among all cases.

\section{ECT bone scan}

Seven patients with preoperative PSA value over $20 \mathrm{ng} / \mathrm{ml}$ received ECT bone scan. During follow-up, patients with PSA recurrence or bone pain also accepted ECT bone scan.

\section{Treatment and follow-up}

Nine patients underwent laparoscopic or robot-assisted radical prostatectomy (RP), among which seven patients whose preoperative PSA value greater than $20 \mathrm{ng} / \mathrm{ml}$ accepted pelvic lymph node dissection, one patient with urethral margin positive underwent adjuvant radiotherapy, and all these nine patients underwent adjuvant hormone therapy (HT). Besides, transurethral resection of the prostate (TURP) was performed in the other one case. All patients acquired complete postoperative follow-up with regular evaluation of abdomen CT scan, gastrointestinal endoscopy, PSA value, testosterone level or ECT bone scan.

\section{Statistical analysis}


All the data were analyzed by Microsoft Excel, with a mean value and standard deviation (SD).

\section{Results}

\section{Characteristics of subjects}

Table 1 showed the general characteristics of the ten cases. From November 2001 to December 2020, there were ten patients diagnosed with PPSRCC in our center, all of whom were male. The average age was 67.8 years (51-79 years). Five patients came to see the doctor because of dysuria. The other five cases had no obvious clinical symptoms and were further examined due to elevated PSA. Nine patients accepted laparoscopic or robot-assisted radical prostatectomy. Only one patient adopted TURP, whose preoperative PSA value was normal and was diagnosed as benign prostate hyperplasia (BPH) before surgery. During the operation, we can visually see the transparent gland tissue lacking blood supply (Fig.1). After surgery, nine patients received adjuvant HT, one of which accepted radiotherapy simultaneously. The other one patient didn't accept any adjuvant therapy. None of the ten cases had gastrointestinal tumor history.

\section{Imaging examination}

All of the ten patients received mpMRI before surgery. mpMRI showed lower signal mass with indistinct margins in the peripheral zone (6/9) and central gland (3/9) of T2-weighted imaging in nine patients. Further in diffusion-weighted imaging, the lesions appeared higher degree of diffusion restriction. PI-RADS score ranged from 4 to 5 in these patients. The mpMRI findings of these nine cases suggested prostate adenocarcinoma, and another one patient was considered to be BPH with PI-RADS score of 2. There were no specific manifestations of SRCC.

\section{Pathological examination}


The diagnosis of PPSRCC is mainly based on pathological diagnosis. Transperineal prostate biopsy was performed in nine patients mentioned above. Among them, five patients were diagnosed as prostatic adenocarcinoma, and the other four cases were found a mixture of SRCC and adenocarcinoma.

Postoperative pathological results confirmed SRCC mixed with prostatic adenocarcinoma in nine cases, and only one patient with pure SRCC (Fig.2). Under light microscopy, signet ring cells were scattered or diffusely infiltrated in the stroma of the prostate in all the ten patients in this group. The cells were round in shape, and the cytoplasm was rich and transparent. The crescent-shaped nuclei were squeezed to one side by vacuoles in the cytoplasm.

After postoperative recovery, colonoscopy and endoscopy were performed in order to exclude metastasis from the gastrointestinal tract to the prostate due to the signet ring cell component. Pancreas and other abdominal organs were evaluated by a CT scan, and no pathological signs were found. Therefore, we considered these cases to be PPSRCC.

\section{ECT results of bone scan}

Seven patients received ECT bone scan preoperatively. Among them, only one case was found suspicious bone metastasis in two ribs, and the other six patients with no signs of bone metastasis. During follow-up, two patients with PSA recurrence or bone pain accepted ECT scan and were found multiple bone metastasis.

\section{Follow-up and prognosis}

During a mean follow up of 43.6 month (7-80 months), all the ten patients accepted regular abdomen CT scan, gastrointestinal endoscopy, PSA value and testosterone level reexamination. Two patients died 18 months and 29 months respectively after surgery because of poor therapeutic response upon HT and further tumor recurrence or multiple bone metastasis. The other eight patients are still in 


\section{Discussion}

SRCCs are most common in the gastrointestinal tract. Therefore, when SRCC is detected in the prostate, endoscopy, colonoscopy, and abdominal CT scan are required to rule out metastasis of gastrointestinal tumors. In this study, no gastrointestinal pathological signs were detected in ten patients, so they were finally diagnosed as PPSRCC. PPSRCC is a kind of rare, poorly differentiated, highly malignant tumor with poor prognosis. Warner et al. statistically reported 29,783 cases of prostate cancer, including 9 cases of PPSRCC [3]. According to their report, the mean onset age was 68 years old, and 6 of them were in clinical stage IV, which had already developed distant metastasis when detected. Views on survival of patients with PPSRCC are divided. Fujita et al. [4] calculated that the 1 -year survival rate of PPSRCC patients was $82 \%$, and the 5-year survival rate was only $11.7 \%$. They suggested that only disease stage at diagnosis was related to the survival rate, but had nothing to do with the serum PSA level nor the treatment method applied. Saito et al. [5] descripted 17 cases of PPSRCC, and found the 5-year survival rate was 0 . Warner et al. showed an average survival of 29 months [3]. In this study, except for the patient who was just followed after surgery for 7 months, 1-year and 5-year survival rate of other patients were $100 \%$ and $77.8 \%$ separately, which were obviously higher than that reported in the literature. We hypothesized that it was probably due to the mixed components of pathological results, including SRCC and adenocarcinoma. It was not difficult to

130 speculate that the smaller the proportion of signet ring cells, the longer the survival time and the better

131 the prognosis of patients. In other word, we hypothesized that if prostatic adenocarcinoma was mixed with SRCC, survival time would be significantly shortened. However, since our sample size is not 
large enough, further studies are needed to verify this hypothesis.

To date, there is no clear diagnostic criteria for PPSRCC. The diagnosis of this disease mainly depends on pathological examination. Epstein [6] believed that the diagnosis could only be established when the signet ring cell component accounted for over $25 \%$ of the whole tumor, and the metastases outside the prostate were excluded. There are many reasons for the signet ring morphology of cells, most of which are due to the formation of intracellular lumen, and the invagination of cancer cell membrane is the basis of the formation of some intracellular lumen. A few of them accumulated in cells due to PSA and PAP. Very few are caused by accumulation of mucus or fat inside the cell. In this study, signet ring cells accounted for more than $30 \%$ of the prostatic tumors, and the mixed prostatic adenocarcinoma was high-grade and poorly differentiated. Postoperative pathological results showed that the Gleason score was more than 8 in seven patients, and most of them were accompanied by nerve infiltration. It is reported that immunohistochemical detection of PPSRCC usually showed positive PSA and PAP and negative CEA in the cytoplasm of cancer cells[5]. Saito et al. [5] stated that the positive rate of PSA and PAP in prostate SRCC cancer tissues was as high as $81.8 \%(9 / 11)$. Kristin et al. [7] reported that the positive rates of PSA and PAP in prostate SRCC cancer tissues were $71.4 \%$ and $66.7 \%$, respectively, while the positive rates of $\mathrm{AB} / \mathrm{PAS}$ and CEA were relatively low. In our study, the positive rate of PSA and PAP were $80 \%(8 / 10)$ and $70 \%$ (7/10) separately, and the negative rate of CEA was $90 \%(9 / 10)$, which were consistent with literature reports.

Because PPSRCC is so rare, there is no standard treatment yet. The ten patients in this group were mainly treated with radical prostatectomy (RP) and hormone therapy (HT). According to the literature, the current treatment for PPSRCC is similar to the traditional treatment for prostate cancer and mainly includes RP, HT, radiotherapy (RT), and chemotherapy. Warner et al [3] believed that the effect of HT 
alone was limited, while HT+RP and HT+RT were more effective. Similarly, in Yasuhiro's report, patients with PPSRCC survived 100 months after initiation of HT+RT without any evidence of tumor progression during their follow-up [8]. Lilleby et al. [9] presented that at 12 months after initiation of HT + RT combination therapy, one of their patients was able to control the disease without distant metastasis. Alpergok et al. [10] reported that a patient who received HT+RT combined treatment also achieved a survival of 16 months without disease progression. Cheng et al. [11] reported 23 PPSRCC cases which were treated with RP+HT and HT+RT. 8 patients died due to tumor metastasis after 6-42 months of survival, 5 patients had elevated PSA after 12-21 months, and 3 patients were lost to follow-up. Therefore, it is not difficult to find that effect of HT+RT or HT+RP therapy is not yet clea. In addition, Roldan et al. [12] have achieved success in chemotherapy of gastrointestinal SRCC. Yasuhiro et al [2] reported a patient with PPSRCC who received chemotherapy with estrogen mustard, docetaxel, carboplatin and other drugs, and died due to liver metastasis 16 months later. Roldan and Yasuhiro considered that chemotherapeutic therapy is also feasible but needs further study [12]. In our study, a total of eight patients were treated with $\mathrm{HT}+\mathrm{RP}$ in the treatment process, among whom one patients passed away due to tumor progression and multiple bone metastasis 29 months after initiation of $\mathrm{RP}+\mathrm{HT}$, and the other seven patients still survived well during follow-up. One patient was treated with $\mathrm{RP}+\mathrm{RT}+\mathrm{HT}$, but the postoperative survival was less than 24 months, probably because of highly malignant prostatic adenocarcinoma. Therefore, we can infer that PPSRCC patients could benefit from radical prostatectomy combined with postoperative endocrine therapy but the mechanism need further study.

$$
\text { Overall, PPSRCC is an extremely rare malignant tumor with few early symptoms, highly }
$$
malignancy, rapid disease progression, poor prognosis and low five-year survival rate. PPSRCC is 
177 frequently accompanied by high grade prostate adenocarcinoma patterns. Early diagnosis and timely

178 radical resection of prostate cancer, endocrine therapy, radiotherapy and chemotherapy and other

179 comprehensive treatment may improve the prognosis of patients. To some extent, several limitations of

180 this paper should be considered. Firstly, the study included a limited number of cases with limited

181 information, and we could not do any comparable analysis. Secondly, patients are regionally

concentrated in China's Jiangsu Province. Therefore, more experience from colleagues in different

regions and medical institutions will contribute to the diagnosis and treatment of PPSRCC and

differentiate it from ordinary prostate adenocarcinoma.

Acknowledgements: This work was supported by the National Natural Science Foundation of China and Technology Association Young Science and Technology Talents Lifting Project.

Authors' contributions: All authors contributed to the study conception and design. Material preparation, data collection and analysis were performed by $\mathrm{Pu} \mathrm{Li}$, Zihan Zhou, and Meiling Bao. The first draft of the manuscript was written by $\mathrm{Pu} \mathrm{Li}$ and all authors commented on previous versions of the manuscript. All authors read and approved the final manuscript.

\section{Declarations}

194 Conflict of Interest: All authors have completed the ICMJE uniform disclosure form. The authors have no conflicts of interest to declare.

Ethical Statement: The authors are accountable for all aspects of the work in ensuring that questions 
in 2013) and this study was approved by the Ethics Committee of the First Affiliated Hospital of Nanjing Medical University (No. 2020-SR-148) and written informed consent for publication of the patients' information and images was entirely obtained.

\section{References}

1. Uyama T, Moriwaki S. Papillary and mucus-forming adenocarcinomas of prostate. Urology $1979 ; 13: 432-4$.

2. Yasuhiro H, Kengo L, Akiko O, et al. An aggressive signet ring cell carcinoma of the prostate in a Japanese man. Case Rep Oncol 2011;4(3):517-20.

3. Warner JN, Nakamura LY, Pacelli A, et al. Primary signet ring cell carcinoma of the prostate. Mayo Clin Proc 2010;85(12):1130-6.

4. Fujita K, Sufao H, Gotoh T, et al. Primary signet ring cell carcinoma of the prostate: report and review of 42 cases. Int J Urol 2004;11(3):178-81.

5. Saito S, Iwaki H. Mucin-producing carcinoma of the prostate: review of 88 cases. Urology $1999 ; 54(1): 141-4$.

6. Epstein JI, Lieberman PH. Mucinous adenocarcinoma of theprostate gland. Am J Surg Pathol $1985 ; 9: 299-308$.

7. Kristin M, Alline MD, Michael B, et al. Signet-ring cell carcinoma of the prostate. Arch Pathol Lab Med 1992;116:99-102.

8. Yoshimura K, Fukui I, Ishikawa Y, et al. Locally-confined signet-ring cell carcinoma of the prostate: A case report of a long-term survivor. Int J Urol 1996;3:406-7.

9. Lilleby W, Axcrona K, Alfsen GC, et al. Diagnosis and treatment of primary signet ring cell 
carcinoma of the prostate. Acta Oncol 2007;46(8):1195-7.

10. Gök A, Tuygun C, Akmansu M, et al. Primary Signet Ring Cell Carcinoma of the Prostate: A Rare Case Report. J Clin Med 2018;7:218.

11. Gu CY, Wu DL, Huang SS, et al. Diagnosis and treatment of signet ring cell carcinoma of the prostate. Chinese Journal of Urology 2009;30(7):487-9.

12. Roldan AM, Nunez NF, Grande E, et al. A primary signet ring cell carcinoma of the prostate with bone metastasis with impressive response to FOLFOX and cetuximab. Clin Genitourin Cancer 2012;10:199-201.

229

Figure legends

Figure 1 Intraoperative appearance of prostatic signet ring cell carcinoma via TURP. Transparent gland tissue lacking blood supply at 7 o'clock (A) and 12 o'clock (B).

Figure 2 Representative histopathology findings of the resected specimen of PPSRCC. (A)

235 staining positively for PSA (magnification $\times 100$ ); (C)Specimen staining positively for PAP

236 (magnification $\times 100)$ 


\section{Figures}
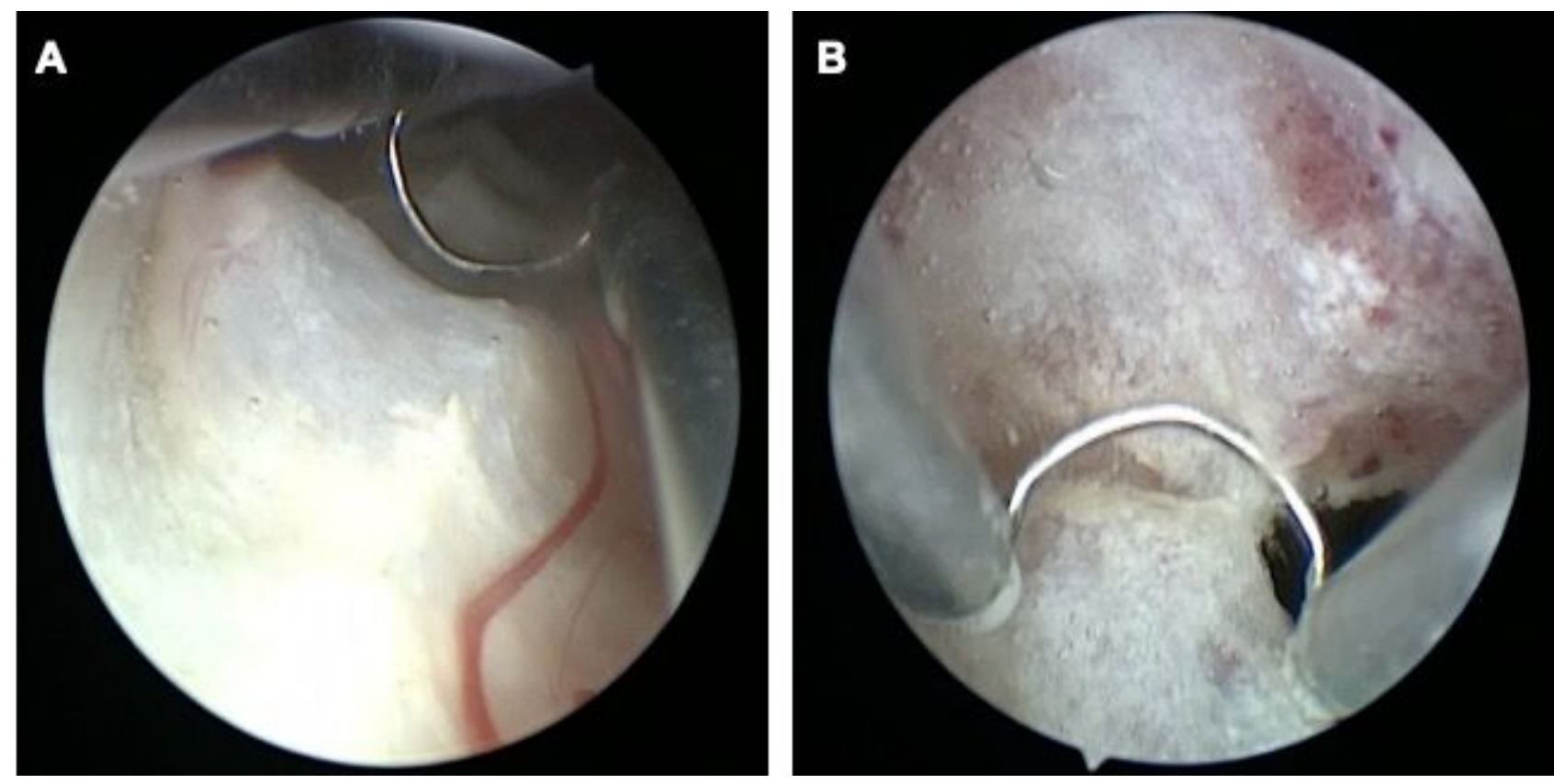

\section{Figure 1}

Intraoperative appearance of prostatic signet ring cell carcinoma via TURP. Transparent gland tissue lacking blood supply at 7 o'clock (A) and 12 o'clock (B).
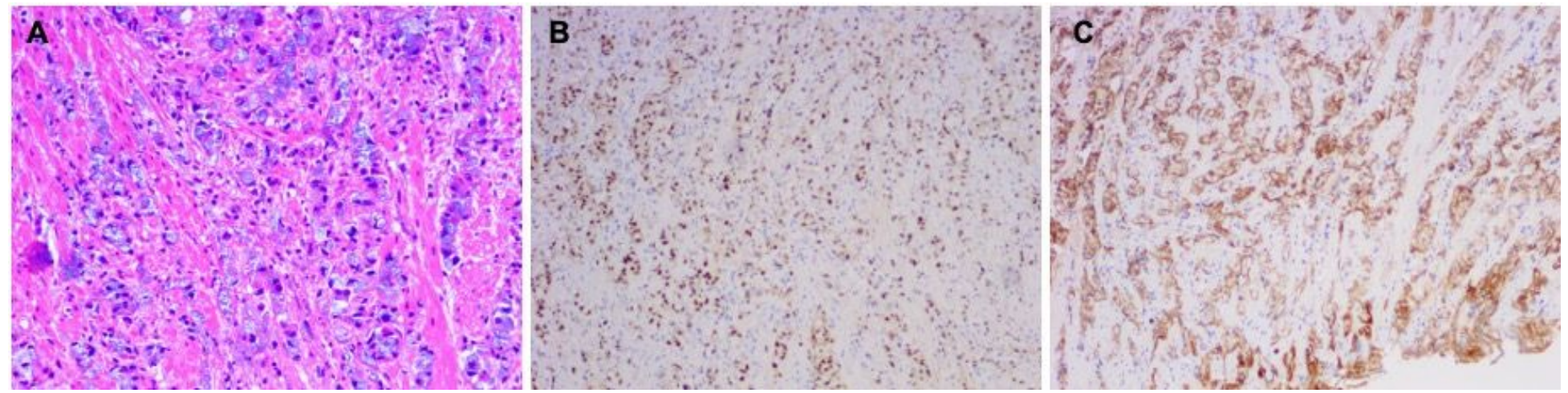

\section{Figure 2}

Representative histopathology findings of the resected specimen of PPSRCC. (A) Specimen with hematoxylin and eosin staining of tumor cells (magnification $\times 200)$; (B)Specimen staining positively for PSA (magnification $\times 100) ;(C)$ Specimen staining positively for PAP (magnification $\times 100$ ) 